VERSION: SEPTEMBER 15, 2021

Preprint typeset using LTEX style emulateapj v. 12/16/11

\title{
UPPER BOUND ON THE FIRST STAR FORMATION HISTORY
}

\author{
Yoshiyuki Inoue $^{1}$, Yasuyuki T. TANaka ${ }^{2}$, Grzegorz M. Madejski ${ }^{1}$ \& Alberto Domínguez ${ }^{3}$ \\ ${ }^{1}$ Kavli Institute for Particle Astrophysics and Cosmology, Department of Physics, Stanford University and SLAC National Accelerator Laboratory, 2575 Sand \\ Hill Road, Menlo Park, CA 94025, USA \\ ${ }^{2}$ Hiroshima Astrophysical Science Center, Hiroshima University, 1-3-1 Kagamiyama, Higashi-Hiroshima, Hiroshima 739-8526, Japan and \\ ${ }^{3}$ Department of Physics \& Astronomy, University of California, Riverside, CA 92521, USA \\ Version: September 15, 2021
}

\begin{abstract}
Our understanding of the nature of the extragalactic background light (EBL) has improved with the recent development of gamma-ray observation techniques. An open subject in the context of the EBL is the reionization epoch, which is an important probe of the formation history of first stars, the so-called Population III (Pop III) stars. Although the mechanisms for the formation of Pop III stars are rather well understood on theoretical grounds, their formation history is still veiled in mystery because of their faintness. To shed light into this matter, we study jointly the gamma-ray opacity of distant objects and the reionization constraints from studies of intergalactic gas. By combining these studies, we obtain a sensitive upper bound on the Pop III star formation rate density as $\dot{\rho}_{*}(z)<0.01[(1+z) /(1+7.0)]^{3.4}\left(f_{\mathrm{esc}} / 0.2\right)^{-1}(C / 3.0) \mathrm{M}_{\odot} \mathrm{yr}^{-1} \mathrm{Mpc}^{-3}$ at $z \geq 7$, where $f_{\text {esc }}$ and $C$ are the escape fraction of ionizing photons from galaxies and the clumping factor of the intergalactic hydrogen gas. This limit is a $\sim 10$ times tighter constraint compared with previous studies that take into account gamma-ray opacity constraints only. Even if we do not include the current gamma-ray constraints, the results do not change. This is because the detected gamma-ray sources are still at $z \leq 4.35$ where the reionization has already finished.
\end{abstract}

Subject headings: stars: Population III - cosmology: dark ages, reionization, first stars - cosmology: diffuse radiation - gamma rays: general

\section{INTRODUCTION}

First stars, the so-called Population III (Pop III) stars, are believed to have played an important role in the early cosmic evolution by emitting intense radiation and by dispersing heavy elements into the interstellar medium (e.g. Bromm et al. 2009). The formation process of Pop III stars has been theoretically studied in detail. Pop III stars were formed when the Universe was less than a few hundred million years old (e.g. Abel et al. 2002) and their typical mass was $\sim 20-40 M_{\odot}$ (Hosokawa et al. 2011, 2012). However, the star formation history of Pop III stars is highly uncertain. The theoretically predicted peak of the star formation rate of Pop III stars varies by four orders of magnitude, over $\sim 10^{-5}-10^{-1} M_{\odot} \mathrm{yr}^{-1} \mathrm{Mpc}^{-3}$ (e.g. Bromm \& Loeb 2006; Tornatore et al. 2007; Johnson et al. 2008; Trenti \& Stiavelli 2009; de Souza et al. 2011). Observationally, it is difficult to directly investigate Pop III stars with current telescopes because Pop III stars are formed at the early stage of the universe's history. Direct measurements will require next generation instruments, as sensitive as the thirty meter telescope 2 and the James Webb Space Telescope ${ }^{3}$.

There are various indirect lines of evidence for Pop III stars - such as reionization (e.g. Totani et al. 2006; Ade et al. 2013). Intense ultraviolet (UV) radiation from Pop III stars was very likely to significantly contribute to the ionization of the intergalactic medium (IGM). Measurements of IGM absorption signatures in the spectra of distant quasars and

E-mail: yinoue@slac.stanford.edu

${ }^{1}$ Pop III stars are divided into Pop III.1 and III.2 (Bromm et al. 2009). Pop III.1 stars are stars formed from cosmological initial conditions, and Pop III.2 stars are zero-metallicity stars but formed from a gas influenced by earlier stars. For simplicity, we treat them as one population.

2 http: / / www.tmt.org

3 http://www. jwst.nasa.gov gamma-ray bursts (GRBs), together with the polarization of the cosmic microwave background $(\mathrm{CMB})$, already prove that the IGM has been reionized between redshift $z \sim 6$ and $\sim 30$ (e.g. Mesinger 2010; Pritchard et al. 2010; Ade et al. 2013).

The Infrared Telescope in Space (IRTS) reported an excess in the NIR background, which can be explained by redshifted light from Pop III stars (Matsumoto et al. 2005, see also Tsumura et al. (2013)). However, the required ionizing photon budget to explain this NIR excess would overproduce the measured Thomson scattering optical depth Madau \& Silk 2005). Later it was also found that this excess would be inconsistent with $\mathrm{TeV}$ observations of nearby blazars (e.g. Aharonian et. al. 2006, but see also Essey \& Kusenko 2010).

Gamma rays propagating through the universe are attenuated by pair production interactions with low-energy photons of the extragalactic background light (EBL; e.g. Gould \& Schréder 1966; Jelley 1966; Dwek \& Krennrich 2013). We can probe the EBL by measuring the attenuation features in the spectra of distant gamma-ray sources (e.g. Aharonian et al. 2006; Ackermann et al. 2012b; Abramowski et al. 2013; Domínguez et al. 2013). UV radiation fields causing the reionization may induce significant gamma-ray absorption above a few tens of $\mathrm{GeV}$ (Oh 2001; Inoue et al. 2010, 2013b).

Based on a galaxy formation model that reproduces the reionization history, it has been shown that it is difficult to distinguish the contribution of Pop III stars through gamma-ray absorption (e.g. Inoue et al. 2013b). On the other hand, without addressing the implications for reionization, Pop III star formation rate density has been constrained through gamma-ray absorption (Raue et al. 2009; Gilmore 2012) or the NIR background (Fernandez \& Komatsu 2006). Shull \& Venkatesan (2008) have investigated the allowed range of Pop III star formation efficiency using the constraints 
from the Thomson scattering optical depth, although it depends on the optical depth of IGM after the reionization.

In this Letter, we present new constraints on the Pop III star formation history, jointly taking account of (1): the Thomson scattering optical depth, (2): neutral fraction of IGM, and (3): observed gamma-ray opacity. Since the NIR background measurement is hampered by uncertain intensity of the zodiacal light, we do not include those constraints. Throughout this Letter, we adopt the standard cosmological parameters of $\left(h, \Omega_{M}, \Omega_{\Lambda}\right)=(0.7,0.3,0.7)$.

\section{CONSTRAINTS ON FIRST STAR FORMATION HISTORY}

Once the star formation history and spectrum of Pop III stars are given, we can derive the Thomson scattering optical depth, neutral fraction, and gamma-ray opacity (see Inoue et al. 2013b, and references therein). Theoretically, the Pop III star formation history $\dot{\rho}_{*}(z)$ has been extensively studied (e.g. Bromm \& Loeb 2006; Tornatore et al. 2007; Johnson et al. 2008; Trenti \& Stiavelli 2009; de Souza et al. 2011). Although all models expect the peak of the Pop III star formation history at $z \gtrsim 6$, the expected history is model dependent. In this Letter, we simply assume the broken powerlaw shape for the Pop III star formation history at redshift $z$ as

$$
\dot{\rho}_{*}(z)=\dot{\rho}_{0}\left[\left(\frac{1+z}{1+z_{\text {peak }}}\right)^{\alpha}+\left(\frac{1+z}{1+z_{\text {peak }}}\right)^{\beta}\right]^{-1},
$$

where $\dot{\rho}_{0}$ gives the normalization in the units of $\mathrm{M}_{\odot} \mathrm{yr}^{-1} \mathrm{Mpc}^{-3}$ and $z_{\text {peak }}$ is the redshift where the star formation rate of Pop III stars is maximum. We investigate the allowed parameter space of $\left(\dot{\rho}_{0}, z_{\text {peak }}, \alpha, \beta\right)$ by comparing against currently available constraints. Since the reionization has occurred at $z \gtrsim 6$, we set $6 \leq z_{\text {peak }} \leq 20$. Even if we set $z_{\text {peak }} \gtrsim 20$, the limit on the Pop III star formation history does not change significantly. We also set $3 \leq \alpha \leq 8$ and $-5 \leq \beta \leq 0$ expecting the presence of a peak in the star formation history. If we set a delta function-like star formation history, we can expect higher star formation rate density.

We follow Inoue et al. (2013b) for the calculation of the Thomson scattering optical depth, neutral fraction, and gamma-ray opacity. In this Letter, we summarize the key assumptions adopted by us. The cosmic stellar emissivity at a given frequency $\nu$ and redshift $z$ is required to evaluate those values and is given by

$$
\begin{aligned}
j(\nu, z)= & \int_{z}^{\infty}\left|\frac{d t}{d z^{\prime}}\right| d z^{\prime} f_{\mathrm{esc}} \dot{\rho}_{*}\left(z^{\prime}\right) \varepsilon\left[\left(1+z^{\prime}\right) \nu /(1+z), z^{\prime}, z\right] \\
& \times \exp \left[-\tau_{\mathrm{IGM}}\left(\nu, z^{\prime}, z\right)\right],
\end{aligned}
$$

where $\varepsilon\left(\nu, z^{\prime}, z\right)$ is the intrinsic emissivity at frequency $\nu$ at $z$ from stars born at $z^{\prime}$ in units of erg s${ }^{-1} \mathrm{~Hz}^{-1} \mathrm{M}_{\odot}^{-1}$ given by stellar population synthesis models and $\tau_{\text {IGM }}$ is the attenuation opacity in the IGM, respectively. We adopt the IGM opacity of Yoshii \& Peterson (1994). $f_{\text {esc }}$ is the escape fraction of photons from galaxies with energy above the threshold for ionization of hydrogen. We use stellar population synthesis models that provide the spectral energy distributions with zero metallicity, namely the model of Schaerer (2003). We adopt the Salpeter initial mass function (Salpeter 1955) for the mass range of 1-100 $M_{\odot}$. Recent radiation-hydrodynamics simulations suggest that the typical mass of Pop III stars is limited to $\lesssim 20-40 M_{\odot}$ due to the radiation feedback effects (Hosokawa et al. 2011, 2012). If there are Pop III stars with different initial mass functions, our limit on the Pop III star formation history will change by a factor equal to the ratio of the integrals of the mass functions. We neglect the interstellar dust extinction effect.

A key parameter is $f_{\text {esc }}$, which we assume here to be 0.2 at all redshifts (Yajima et al. 2011), unless we note otherwise. Observationally, $f_{\text {esc }} \simeq 0.05-0.3$ is reported at $z \sim 3$ (Nestor et al. 2013), but values at $z \geq 4$ have not been measured yet. Ono et al. (2010) have set upper limits of $f_{\mathrm{esc}} \lesssim 0.6$ at $z=5.7$ and $f_{\text {esc }} \lesssim 0.9$ at $z=6.6$ for LAEs. Gong \& Cooray (2013) independently constrained $f_{\text {esc }}$ as $\sim 0.5$ at $z=3$ and $\sim 0.9$ at $z=6$ using constraints on gamma-ray opacity. Another key parameter to evaluate the reionization is the clumping factor $C=\left\langle n_{H}^{2}\right\rangle / \bar{n}_{H}^{2}$, where $n_{\mathrm{H}}$ is the hydrogen density (see Inoue et al. 2013b, for details). At $z \gtrsim 6, C$ is constrained as $\lesssim 3$ by combining hydrodynamical simulations and the measurements of the Ly $\alpha$ forests (Bolton \& Haehnelt 2007). We take $C=3.0$ as the fiducial value of the parameter unless otherwise noted.

\subsection{Constraint from the Thomson scattering optical depth}

During the reionization epoch, a large population of free electrons is generated in the IGM. Those electrons scatter the CMB photons, and their optical depth can be estimated from the CMB measurements. The measured electron Thomson scattering optical depth $\tau_{e}$ is $\tau_{e}=0.092 \pm 0.013$ inferred by the Planck data (Ade et al. 2013). Since Population I and II stars also contribute to the reionization, our model must not overproduce the free electron content solely with Pop III stars.

\subsection{Constraint from the neutral fraction of hydrogen}

As the universe was being reionized, the neutral fraction of intergalactic hydrogen $x_{\mathrm{HI}}$ also changed. $x_{\mathrm{HI}}$ is a good tracer of the ionizing photon production history during the reionization epoch. The discovery of broad troughs of Ly $\alpha$ absorption, the so-called Gunn-Peterson troughs Gunn \& Peterson 1965), in the spectra of distant objects have enabled an estimate of the neutral fraction as $x_{\mathrm{HI}} \geq 0.033$ at $z=6.2$ (Mesinger \& Haiman 2007), $x_{\mathrm{HI}} \geq 0.1$ at $z=7.08$ (Mortlock et al. 2011), and $x_{\mathrm{HI}} \leq 0.17$ at $z=6.3$ (Totani et al. 2006).

More ionizing photons and lower neutral fraction are expected, if we take into account the Population I and II stars. Therefore, we constrain the Pop III star formation history not to be less than the lower limit of the neutral fraction. We have not attempted a detailed comparison with Gunn-Peterson measurements of quasars at $z \lesssim 6$. Such effects depend rather sensitively on the dense region of the IGM gas, which is not essential for studying the Pop III star formation at $z \gtrsim 6$.

\subsection{Constraint from the gamma-ray attenuation}

Sufficient UV radiation fields causing cosmic reionization may induce gamma-ray absorption above a few tens of $\mathrm{GeV}$ (Oh 2001; Inoue et al.2010, 2013b). To constrain the gammaray opacity at high redshifts, we use the Fermi-LAT detected distant sources with detected $>10 \mathrm{GeV}$ photons. Since gamma-ray opacity for photons with $\sim 20-70 \mathrm{GeV}$ is close to unity at $z \gtrsim 2$ (e.g. Domínguez et al. 2011; Inoue et al. 2013b), we focus on sources at $z \geq 2$.

Known distant gamma-ray emitting populations are GRBs and blazars. LAT has detected 35 GRBs Ackermann et al. 2013). Since more distant sources can put tighter constraints on the Pop III star formation rate, we use the most distant one GRB 080916C at $z=4.35$, which had photons up 
to $13.22 \mathrm{GeV}$. We select blazars having Test Statistics (TS) $>100$, photon index $\Gamma \leq 2.0$, and $z>2.0$ from the second catalog of Fermi-LAT sources (2FGL Nolan et al. 2012). After the selection, flat spectrum radio quasar 2FGL J1344-1723 at $z=2.506$ survives with $\mathrm{TS}=967$.

For both the GRB and blazar cases, we reduced FermiLAT data and performed an unbinned maximum likelihood analysis using Fermi Science Tool version v9r27p1. For 2FGL J1344-1723, the analyzed time interval is MET 239557417 (2008-08-04 15:43:36 UT) to 386632050 (2013-04-02 21:47:27 UT) representing a total of $\sim 4.7$ years of data. For GRB 080916C, we used the first 1000 seconds after the burst 4 (MET 243216766-243217766). We extracted $0.1-300 \mathrm{GeV}$ SOURCE class events within $10^{\circ}$ circles centered at the source positions. Good time intervals were generated using the recommended filter of $($ DATA_QUAL $==1) \& \&($ LAT_CONFIG $==1) \& \& A B S(R O C K$ _ANGLE) $<52$ and an ROI-based zenith angle cut (roicut=yes) with a maximum zenith angle cut of $100^{\circ}$. We utilized P7SOURCE_V6 as the Instrument Response Functions. In the source model of 2FGL J1344-1723, we modeled the spectrum as a log-parabola and included all the 2 FGL sources within a $10^{\circ}$ circle. The spectral parameters of these sources were left free in the likelihood fitting. 2FGL sources within an annulus of $10^{\circ}$ to $15^{\circ}$ from the source were also included in the model, but their spectral parameters were fixed to the 2FGL values. In the source model of GRB 080916C, we included only this burst by modeling its spectrum with a single power-law function. Galactic and extragalactic diffuse emissions were modeled with the recommended template files (gal_2yearp7v6_v0.fits and iso_p7v6source.txt), where the normalizations of both components were set free and the standard power-law scaling was applied to the Galactic one. As a result, we obtained a photon index of $\alpha=2.08 \pm 0.06$ and a curvature parameter $\beta=0.10 \pm 0.03$ for $2 \mathrm{FGL} \mathrm{J} 1344-1723$ and $\Gamma=2.18 \pm 0.08$ for GRB 080916C. To construct the LAT spectrum, we divided the whole energy range into bins defined by energies of $0.1-0.3,0.3-1.0,1.0-3.0,3-10,10-30$, and $30-100 \mathrm{GeV}$ and performed a maximum-likelihood analysis using gtlike in each energy bin. We assumed single power-law shapes for both sources allowing the normalization to vary. Here the power law index was fixed to the values obtained by gt like from the whole energy-range $(0.1-300 \mathrm{GeV})$ data set for GRB 080916C, while we utilize the $\alpha$ value of the log-parabola spectrum for 2FGL J13441723. For the surrounding sources and the two (Galactic and isotropic) diffuse components, the spectral parameters were again fixed to the values obtained by gt like for the whole energy range and only the normalizations were left free. The estimated systematic uncertainty on the $\gamma$-ray flux is $10 \%$ at $100 \mathrm{MeV}$, decreasing to $5 \%$ at $560 \mathrm{MeV}$, and increasing to $10 \%$ at $10 \mathrm{GeV}$ and above (Ackermann et al. 2012a).

To constrain the gamma-ray opacity, an intrinsic spectrum is required. There are various models to explain blazar and GRB spectra. We follow general arguments based on the shock acceleration scenario. First-order Fermi acceleration is the broadly accepted model, and the resulting particle spectrum is expected to show a power-law shape of $d N / d E \propto E^{-p}$ with a slope $p \sim 2$. The slope $p$ can be steeper than 2 due to cooling effects. The expected photon index from the inverse Compton scattering radiation by electrons will be $\Gamma \geq$

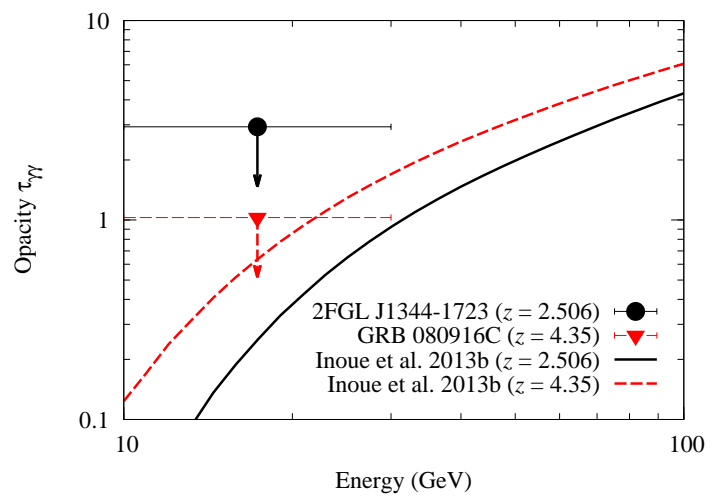

FIG. 1.- Derived upper limits for the optical depth at $z=2.506$ (2FGL J1344-1723, solid arrow) and, $z=4.35$ (GRB 080916C, dashed arrow). For the comparison, we also plot an expected optical depth from an EBL model by curves (Inoue et al. 2013b).

$(1+p) / 2$, while that from hadronic processes will be $\Gamma=p$, where the photon spectrum is given by $d N_{\gamma} / d E \propto E^{-\Gamma}$. The resulting photon index of the intrinsic spectrum is $\Gamma \geq 1.5$ (e.g. Aharonian et al. 2006). If we assume a softer intrinsic spectrum, we can obtain a tighter constraint on Pop III star formation rate density. To be conservative, we take $\Gamma=1.5$ (e.g. Aharonian et al. 2006) from the energy bin of $3.0-10.0 \mathrm{GeV}$, which is the highest energy bin among the data points not affected by the gamma-ray attenuation. The gamma-ray opacity at energy $E$ and redshift $z$ is estimated as $\tau_{\gamma \gamma}(E, z)=\ln \left[F_{\text {int }}(E) / F_{\text {obs }}(E)\right]$, where $F_{\text {int }}$ is the intrinsic flux and $F_{\text {obs }}$ is the observed flux. We take this opacity as an upper limit. Figure 1 shows the derived opacity limit for 2FGL J1344-1723 and GRB 080916C. The derived upper limit at $E=17.3 \mathrm{GeV}$ is $\tau_{\gamma \gamma} \leq 2.93$ and $\leq 1.03$ at $z=2.506$ and $z=4.35$, respectively. We also show the opacity from the model of Inoue et al. (2013b) for reference. We note that hadron-induced gamma rays may appear above several hundred GeV (Essey \& Kusenko 2010).

In summary, constraints on Pop III stars obtained by current available observations are $\tau_{e} \leq 0.092$ (Ade et al. 2013), $x_{\mathrm{HI}} \geq 0.033$ at $z=6.2$ (Mesinger \& Haiman 2007), $x_{\mathrm{HI}} \geq 0.1$ at $z=7.08$ (Mortlock et al. 2011), $\tau_{\gamma \gamma} \leq 2.93$ at $E=17.3 \mathrm{GeV}$ and $z=2.506$, and $\tau_{\gamma \gamma} \leq 1.03$ at $E=17.3 \mathrm{GeV}$ and $z=4.35$. Since probability functions of these limits are uncertain, we set the upper limits by taking the maximum allowed star formation rate density at each redshift within each allowed parameter spaces. Therefore, first, we estimate $\tau_{e}, x_{\mathrm{HI}}$ and $\tau_{\gamma \gamma}$ in all the combinations of parameters. Second, we choose parameter sets that do not violate the given observational constraints. Lastly, we take the maximum of the star formation rate density at each redshift from the allowed parameter sets and set those as the upper limit on the Pop III star formation history.

\section{RESULTS}

Figure 2 shows the upper limits on the Pop III star formation history. Thick solid, dashed, and dotted curves with arrows show the upper limit on the Pop III star formation rate density for $\left(C, f_{\mathrm{esc}}\right)=(3.0,0.2),(3.0,0.5)$, and $(1.0,0.2)$, respectively. The upper limit above $z=7$ can be approximated as

$$
\dot{\rho}_{*}(z)<0.01\left[\frac{(1+z)}{(1+7.0)}\right]^{3.4}\left(\frac{f_{\text {esc }}}{0.2}\right)^{-1}\left(\frac{C}{3.0}\right) \mathrm{M}_{\odot} \mathrm{yr}^{-1} \mathrm{Mpc}^{-3} .
$$




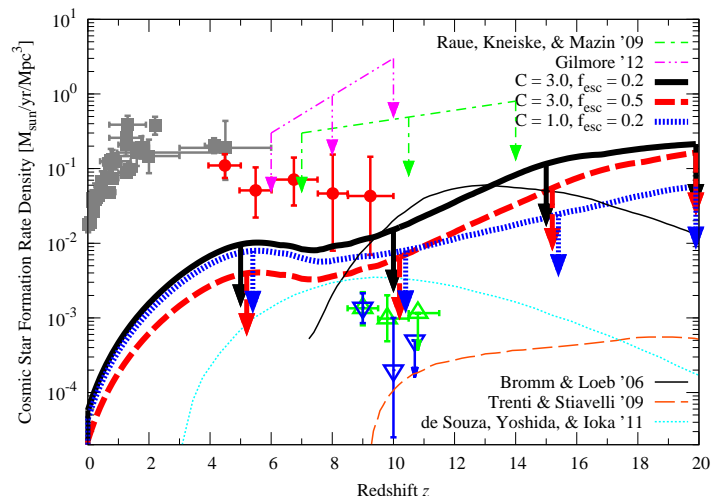

FIG. 2.- Upper limits on the Pop III star formation history. Thick solid, dashed, and dotted curve with arrows represents the upper limit for $\left(C, f_{\text {esc }}\right)=$ $(3.0,0.2),(3.0,0.5)$, and $(1.0,0.2)$, respectively. For comparison, we show the previously studied upper limits by Raue et al. (2009) and Gilmore (2012) with thin dot-dashed line and double-dot-dashed line with arrows. We plot theoretical models for the Pop III star formation by Bromm \& Loeb (2006), thin solid curve; Trenti \& Stiavelli (2009) (Pop III.2), dashed curve; and de Souza et al. (2011) (Pop III.2), dot-dashed curve. For de Souza et al. (2011), we adopt their optimistic model. We also plot the observational data compiled by Hopkins (2004, filled square), that deduced from high redshift galaxies (Ellis et al.|2013; Oesch et al.|2013, up-triangle, and down-triangle, respectively), and that inferred from GRBs (Kistler et al. 2013, circle). These data points represent the summation of all stellar populations. For the high redshift galaxies, limiting luminosities are adopted by each author.

For comparison, we show the upper-limits from previous studies (Raue et al. 2009; Gilmore 2012), which do not take into account the reionization constraints. The upper limit becomes 10 times tighter by including the reionization data. The upper limit does not change significantly when we exclude the constraints from the Fermi-LAT data. This is because the most distant gamma-ray objects with $>10 \mathrm{GeV}$ photons are still not beyond $z \sim 6$ where the reionization occurred.

We also plot the cosmic star formation rate density data compiled by Hopkins (2004), deduced from distant galaxies (Ellis et al. 2013; Oesch et al. 2013), and inferred from GRBs (Kistler et al.2013). We note that these data represent all stellar populations. Each high redshift galaxy data point is obtained by integrating its luminosity function down to a limiting luminosity. We note that the overall UV luminosity function at these redshifts is still uncertain due to poor statistics (Ellis et al. 2013; Oesch et al. 2013).

We compare our limits with theoretically studied Pop III star formation rates in the literature, specifically in Bromm \& Loeb (2006, Pop III.1 + III.2), Trenti \& Stiavelli (2009, Pop III.2), and de Souza et al. (2011, Pop III.2). For de Souza et al. (2011), we take their optimistic model where they assume a very high star formation efficiency and low chemical enrichment. The Pop III star formation model by Bromm \& Loeb (2006) violates the current observational constraints.

If remnants of Pop III stars became seeds of supermassive black holes (SMBHs), which would manifest themselves as quasars observed at $z \sim 6$ (e.g. Mortlock et al. 2011), the presence of such quasars may tighten our limits. In this Letter, we do not take into account this constraint because of our poor knowledge of accretion and merger history of such seed BHs, and their precursors. It may be difficult to create SMBHs from Pop III stars because radiation feedback terminates Pop III stars growing above a few tens solar masses (e.g. Hosokawa et al. 2011). Promising candidates for seeds of SMBHs can be formed by a direct collapse of supermassive stars (Hosokawa et al. 2013). Since their effective temperature is less than $10^{4} \mathrm{~K}$ because of large radius of such supermassive stars, they will not significantly contribute to the reionization.

\section{CONCLUSIONS}

In this Letter, we show that considering simultaneously the current measurements of gamma-ray opacity, the electron Thomson scattering optical depth, and the neutral fraction set an upper limit of $\dot{\rho}_{*}(z)<0.01[(1+$ $z) /(1+7.0)]^{3.4}\left(f_{\text {esc }} / 0.2\right)^{-1}(C / 3.0) \quad \mathrm{M}_{\odot} \quad \mathrm{yr}^{-1} \mathrm{Mpc}^{-3}$ on the Pop III star formation rate density above $z=7$. By including the reionization constraints, the upper limit becomes $\sim 10$ times tighter compared to previous works (Raue et al. 2009; Gilmore 2012).

Current gamma-ray data do not strongly constrain the Pop III star formation history because the most distant object is still at $z=4.35$ where the reionization process is completed. To explore the reionization epoch via the gamma-ray technique, sources beyond redshift 6 and rich with $>10 \mathrm{GeV}$ photons would be needed (Inoue et al. 2013b). Fermi may eventually detect blazars at $z>6$ (Inoue et al. 2011), and the Cherenkov Telescope Array may possibly do the same at $\sim 30$ $\mathrm{GeV}$ for GRBs (Inoue et al. 2013a). Tanaka et al. (2013) have reported the detection of $>100 \mathrm{GeV}$ photons from a blazar at $z=1.1$ and Takahashi et al. (2013) have reported a candidate Fermi gamma-ray blazar at $z \sim 3-4$. Future gamma-ray observations of distant sources at $\gtrsim 10 \mathrm{GeV}$ will put tighter constraints on Pop III star formation history by combining reionization measurements.

We acknowledge M. Ajello, J. Chiang, L. Latronico, P. Marshall, J. Perkins, J. Scargle, and D. Thompson for useful comments. The Fermi-LAT Collaboration acknowledges support from a number of agencies and institutes for both development and the operation of the LAT as well as scientific data analysis. These include NASA and DOE in the United States, CEA/Irfu and IN2P3/CNRS in France, ASI and INFN in Italy, MEXT, KEK, and JAXA in Japan, and the K. A. Wallenberg Foundation, the Swedish Research Council and the National Space Board in Sweden. Additional support from INAF in Italy and CNES in France for science analysis during the operations phase is also gratefully acknowledged.

\section{REFERENCES}

Abel, T., Bryan, G. L., \& Norman, M. L. 2002, Science, 295, 93

Abramowski, A. et al. 2013, A\&A, 550, A4

Ackermann, M. et al. 2012a, ApJS, 203, 4

- 2012b, Science, 338, 1190

-. 2013, arXiv:1303.2908

Ade, P. A. R. et al. 2013, arXiv:1303.5076

Aharonian, F. et al. 2006, Nature, 440, 1018

Bolton, J. S. \& Haehnelt, M. G. 2007, MNRAS, 382, 325

Bromm, V. \& Loeb, A. 2006, ApJ, 642, 382
Bromm, V., Yoshida, N., Hernquist, L., \& McKee, C. F. 2009, Nature, 459, 49

de Souza, R. S., Yoshida, N., \& Ioka, K. 2011, A\&A, 533, A32

Domínguez, A., Finke, J. D., Prada, F., Primack, J. R., Kitaura, F. S., Siana,

B., \& Paneque, D. 2013, ApJ, 770, 77

Domínguez, A. et al. 2011, MNRAS, 410, 2556

Dwek, E. \& Krennrich, F. 2013, Astroparticle Physics, 43, 112

Ellis, R. S. et al. 2013, ApJ, 763, L7

Essey, W. \& Kusenko, A. 2010, Astroparticle Physics, 33, 81 
Fernandez, E. R. \& Komatsu, E. 2006, ApJ, 646, 703

Gilmore, R. C. 2012, MNRAS, 420, 800

Gong, Y. \& Cooray, A. 2013, ApJ, 772, L12

Gould, R. J. \& Schréder, G. 1966, Physical Review Letters, 16, 252

Gunn, J. E. \& Peterson, B. A. 1965, ApJ, 142, 1633

Hopkins, A. M. 2004, ApJ, 615, 209

Hosokawa, T., Omukai, K., Yoshida, N., \& Yorke, H. W. 2011, Science, 334, 1250

Hosokawa, T., Yorke, H. W., Inayoshi, K., Omukai, K., \& Yoshida, N. 2013, ApJ, 778, 178

Hosokawa, T., Yoshida, N., Omukai, K., \& Yorke, H. W. 2012, ApJ, 760, L37

Inoue, S., Salvaterra, R., Choudhury, T. R., Ferrara, A., Ciardi, B., \& Schneider, R. 2010, MNRAS, 404, 1938

Inoue, S. et al. 2013a, Astroparticle Physics, 43, 252

Inoue, Y., Inoue, S., Kobayashi, M. A. R., Makiya, R., Niino, Y., \& Totani, T. 2013b, ApJ, 768, 197

Inoue, Y., Inoue, S., Kobayashi, M. A. R., Totani, T., Kataoka, J., \& Sato, R. 2011, MNRAS, 411, 464

Jelley, J. V. 1966, Physical Review Letters, 16, 479

Johnson, J. L., Greif, T. H., \& Bromm, V. 2008, MNRAS, 388, 26

Kistler, M. D., Yuksel, H., \& Hopkins, A. M. 2013, arXiv:1305.1630

Madau, P. \& Silk, J. 2005, MNRAS, 359, L37

Matsumoto, T. et al. 2005, ApJ, 626, 31

Mesinger, A. 2010, MNRAS, 407, 1328

Mesinger, A. \& Haiman, Z. 2007, ApJ, 660, 923
Mortlock, D. J. et al. 2011, Nature, 474, 616

Nestor, D. B., Shapley, A. E., Kornei, K. A., Steidel, C. C., \& Siana, B. 2013, ApJ, 765, 47

Nolan, P. L. et al. 2012, ApJS, 199, 31

Oesch, P. A. et al. 2013, arXiv:1301.6162

Oh, S. P. 2001, ApJ, 553, 25

Ono, Y., Ouchi, M., Shimasaku, K., Dunlop, J., Farrah, D., McLure, R., \& Okamura, S. 2010, ApJ, 724, 1524

Pritchard, J. R., Loeb, A., \& Wyithe, J. S. B. 2010, MNRAS, 408, 57

Raue, M., Kneiske, T., \& Mazin, D. 2009, A\&A, 498, 25

Salpeter, E. E. 1955, ApJ, 121, 161

Schaerer, D. 2003, A\&A, 397, 527

Shull, J. M. \& Venkatesan, A. 2008, ApJ, 685, 1

Takahashi, Y., Kataoka, J., Niinuma, K., Honma, M., Inoue, Y., Totani, T., Inoue, S., Nakamori, T., \& Maeda, K. 2013, arXiv:1306.3552

Tanaka, Y. T. et al. 2013, arXiv:1308.0595

Tornatore, L., Ferrara, A., \& Schneider, R. 2007, MNRAS, 382, 945

Totani, T., Kawai, N., Kosugi, G., Aoki, K., Yamada, T., Iye, M., Ohta, K., \& Hattori, T. 2006, PASJ, 58, 485

Trenti, M. \& Stiavelli, M. 2009, ApJ, 694, 879

Tsumura, K., Matsumoto, T., Matsuura, S., Sakon, I., \& Wada, T. 2013, arXiv: 1307.6740

Yajima, H., Choi, J.-H., \& Nagamine, K. 2011, MNRAS, 412, 411

Yoshii, Y. \& Peterson, B. A. 1994, ApJ, 436, 551 